\title{
Pricing the risk-transfer financial instruments via Monte Carlo methods
}

Maciej Romaniuk (mroman@ibspan.waw.pl)

\section{Approved by \\ Yuri Ermoliev (ermoliev@iiasa.ac.at) \\ Institute Scholar, IIASA}

September 2002 


\begin{abstract}
The paper is devoted to finding the present value of catastrophe bonds using a combination of Monte Carlo and Iterative Stochastic Equation methods. Apart from general methodology, three practical examples of catastrophe bonds connected with earthquakes are also considered. For these examples algorithms in pseudocode with procedures originated from catastrophe simulation software are provided. The methodology presented in this paper may be also used for other types of risk-transfer financial instruments. Some of these possibilities are described.

Keywords: catastrophe bond, present value, Monte Carlo method, stochastic equation, pseudocode algorithm, Brownian motion
\end{abstract}




\section{Acknowledgments}

I would like to thank Professor Yuri Ermoliev from IIASA's Risk, Modelling and Society Project for helpful advices and guidance during the Young Scientists Summer Program, and also Dr. Keith Compton and Dr. Koko Warner for their help.

I would like also to thank the Polish Member Organization and IIASA for support during the Young Scientists Summer Program. 


\section{Contents}

1 Introduction $\quad 1$

2 Significant example $\quad 2$

3 Preliminaries and basic assumptions $\quad 4$

3.1 Process of underlying asset movements . . . . . . . . . . . . . . 4

3.1.1 Method of Iterative Stochastic Equation . . . . . . . . . . . 4

3.1.2 Geometrical Wiener process . . . . . . . . . . . . . 6

3.1.3 Other types of stochastic processes . . . . . . . . . 7

3.2 Monte Carlo methods for pricing financial instruments . . . . . . . . 8

3.2 .1 Antithetic variables method . . . . . . . . . . . . 9 9

3.3 Catastrophe bonds . . . . . . . . . . . . . . . . . 10

3.4 EDGE software . . . . . . . . . . . . . . . . . 12

4 General outline $\quad \mathbf{1 2}$

4.1 Application to cat bonds pricing . . . . . . . . . . . . . 13

5 Examples 14

5.1 Introductory example . . . . . . . . . . . . . . . . . . . 14

5.2 Cat bonds depending on magnitude . . . . . . . . . . . 16

5.3 Cat bonds depending on amount of losses . . . . . . . . . . . . . . . 19

6 Concluding remarks $\quad 23$ 


\title{
Pricing the risk-transfer financial instruments via Monte Carlo methods
}

\author{
Maciej Romaniuk (mroman@ibspan.waw.pl)*
}

\section{Introduction}

During the last two decades the losses from natural and human-made catastrophes increased significantly (see e.g. [19]). For example, between 1989 and 1995 total insured losses were $50 \%$ higher than in the preceding 38 years (see [15]). The direct damages from natural disasters have increased nine-fold within the last three decades (see [4]). For many countries, even developed ones, losses caused by these events considerably affected insurance industries, national economy and governmental budgets.

The increasing amount of losses from catastrophes triggered ideas for developing new financial instruments - catastrophe bonds, in abbreviation cat bonds. These instruments are able to transfer losses to financial markets. The catastrophe bond is very similar to normal bond issued by a government or an enterprise. The main difference is that the structure of payments of the catastrophe bond depends on the occurrence of specified types of natural catastrophe in the specific region and time interval. This event defines so called triggering point, which changes the schedule of future payments of the cat bond (see [15], [17], [23], [25], [28]). Examples of catastrophes may be floods, earthquakes, hurricanes, etc.

The cat bond may have the following structure of payments: if there is no earthquake in California till the end of this year, the purchaser of the bond will receive $\$ 5$. Otherwise he will receive only $\$ 1$.

The catastrophe bonds may be a very important new financial instruments for insurers and governments. Especially in the developing countries these instruments may stabilize economic growth. In developed countries they may be also significant for national economy and private sector (see e.g. [3], [10], [25]). These instruments transfer risk from insurance markets or governmental budgets to financial markets, which are more liquid and have more capacity than other markets or budgets (see e.g. [6], [12], [15], [17]).

An important problem is to evaluate the price for cat bonds. From the above example, it is easily seen that this price depends on frequency of earthquakes in California. For other bonds the price may depend also on the movements of interest rates during the specified year.

\footnotetext{
* Systems Research Institute Polish Academy of Sciences, ul. Newelska 6, 01-447 Warszawa, Poland
} 
As we have seen from previous simple example, for cat bonds we always know the structure of payments. However, one of the most important and complex issues is finding the answer for very simple question - how much should we pay now for this financial instrument? In the language of economics what is the present value of catastrophe bond?

This paper is concerned solely with a problem of finding a fair price, not with the demand of this instrument or its attractiveness for sellers or purchasers. The latter depends very much on the specific case, e.g. availability of other risk management instruments (see e.g. [31]).

We will use a combination of Iterative Stochastic Equation (in abbreviation ISE) and Monte Carlo (in abbreviation MC) methods for pricing cat bonds (see e.g. [16], [30], [32], [34]). As we will see, these methods are very flexible and universal. In this paper we present a general outline and three practical examples. These examples deal with pricing of a catastrophe bond connected with an earthquake. However, as we will see, linkage of ISE and Monte Carlo methods may be used for variety of cat bonds and many kinds of processes of underlying asset movements - e.g. cat bonds for floods, hurricanes, etc.

The pricing of cat bonds need inputs from special software generating possible scenarios of catastrophes. We use in this paper EDGE software developed at IIASA (see [1], [33]). However, the other prospects are possible, e.g. the use of flood generator for Upper Tisza River (see [8]).

This paper is organized as follows. Section 2 presents a simple but important introductory example of cat bond pricing. Section 3 provides the necessary preliminaries and some basic assumptions: an introduction to the ISE method, an introduction to the Monte Carlo method for pricing financial instruments, description of catastrophe bonds, and the software for earthquake simulation.

Section 4 presents a general approach for pricing financial instruments using of ISE and MC methods. The specific issue of pricing cat bonds is described in Section 4.1. Section 5 is completely devoted to three examples: the introductory example, cat bonds depending on magnitude of earthquake and cat bonds depending on cumulated amount of losses from earthquakes. Section 6 provides final conclusions and discussion about perspectives for further research.

\section{Significant example}

In this section we present a very simple illustrative example. It illustrates problems connected with the pricing of a catastrophe bond. We will return to this example once again in Section 5.1.

Suppose that we are to find a price for a cat bond. The payment from this bond will occur in future and it depends on two processes. The first process is the behaviour of interest rates on the given market. Suppose that the geometrical Brownian motion (see Section 3.1.2) models this process. In the following we will use symbol $\mathcal{S}_{t}$ and refer to it as the process of underlying asset movements. For example the catastrophe bond considered in this Section may be written on the index of the market.

The second process is connected with the specific nature of cat bonds (see Section 
3.3), i.e. changing of the structure of payments. Assume if there is a catastrophe till the time $T$, purchaser of our instrument will receive no payment. In the other case, he will receive a payment at time moment $T$. The quantity of this payment equals the full price of the market index at $T$. Let us denote this amount as $\mathcal{S}_{T}$.

The time of catastrophe occurrence is a random variable $\mathcal{X}$. Suppose it is modelled by exponential distribution with intensity $\lambda$, i.e.

$$
\operatorname{Pr}(\mathcal{X} \leq x)= \begin{cases}0 & \text { if } x<0 \\ 1-e^{-\lambda x} & \text { otherwise }\end{cases}
$$

where $\operatorname{Pr}(\mathcal{X} \leq x)$ is the probability that catastrophe occurs before time moment $x$.

We also assume that the process of movements of underlying asset $\mathcal{S}_{t}$ and time of catastrophe $\mathcal{X}$ are independent variables.

The payment of this cat bond can be written in the following functional form

$$
f\left(\mathcal{S}_{t}, \mathcal{X}\right)= \begin{cases}0 & \text { if } \mathcal{X} \leq T \\ \mathcal{S}_{T} & \text { otherwise }\end{cases}
$$

Let $C$ denote the price for such a catastrophe bond and $r$ be a risk-free banking yield. The price $C$ is also called a present value of the bond. A standard way to define $C$ is to use form of expected value of cash flow (see e.g. [22], [34]). In this paper we will not consider other possibilities, e.g. to define $C$ as some quantiles. However, this will not affect general approach presented here.

It is easy to see that

$$
\begin{aligned}
C=\mathbb{E}\left(e^{-r T} f\left(\mathcal{S}_{t}, \mathcal{X}\right)\right)=\mathbb{E}\left(e^{-r T} f\left(\mathcal{S}_{t}, \mathcal{X}\right) \mid \mathcal{X} \leq T\right) \operatorname{Pr}(\mathcal{X} \leq T)+ \\
+\mathbb{E}\left(e^{-r T} f\left(\mathcal{S}_{t}, \mathcal{X}\right) \mid \mathcal{X}>T\right) \operatorname{Pr}(\mathcal{X}>T)=e^{-r T} \mathbb{E}\left(\mathcal{S}_{T}\right) \operatorname{Pr}(\mathcal{X}>T)
\end{aligned}
$$

where $\mathbb{E}$ denotes the expected value of random variable and $e^{-r T}$ is a so-called discounting factor for cash flow at time moment $T$ (see e.g. [21]). In equation (3) we used the assumption that $\mathcal{S}_{T}$ and $\mathcal{X}$ are independent random variables and the formula (2).

It is easy to see that quantities $\mathbb{E}\left(\mathcal{S}_{T}\right)$ and $\operatorname{Pr}(\mathcal{X}>T)$ in this simple example can be presented in explicit form. The average value of stochastic process $\mathcal{S}_{t}$ at time moment $T$ can be calculated by using stochastic calculus, i.e. Ito integral (see e.g. [34]). The tail probability $\operatorname{Pr}(\mathcal{X}>T)$ for random variable $\mathcal{X}$ can be calculated by the standard calculus of integrals.

However, this can be done only because we deal with very simple stochastic process $\mathcal{S}_{t}$ and random variable $\mathcal{X}$. If the distribution of $\mathcal{X}$ is more complicated than in (1), the calculation of the appropriate tail probability may be analytically intractable. We have the same situation if the payment function $f\left(\mathcal{S}_{t}, \mathcal{X}\right)$ is more complicated or it depends on additional properties of catastrophe process. For example, there are catastrophe bonds which depend not only on time moment of the first catastrophe, but also on its magnitude (e.g. for earthquakes) or amount of losses caused by catastrophe (see Section 3.3). In such cases the equation similar to (3) may not be hold or become too much complicated for direct calculations. These appear very often in real-life applications. 
For these cases there is a need for other methods of pricing cat bonds. A very important and fruitful approach is to apply the Monte Carlo method. In next Sections we will develop a general methodology and use it for a problem of pricing of complex cat bonds.

\section{Preliminaries and basic assumptions}

\subsection{Process of underlying asset movements}

As it was mentioned in Section 2, the process of movements of the underlying asset (e.g. indexes, prices of stocks, interest rates) may be modelled by some stochastic process. A classical example of such a process is the geometrical Wiener process (or geometric Brownian motion). This stochastic process is often used for pricing the financial instruments. A well known result is the Black-Scholes formula for a calculating the price of European-style options (see e.g. [22]).

The idea of using geometrical Brownian motion was a serious breakthrough in financial mathematics. A variety of papers are devoted to the discussion of this innovation. It was discovered that many financial markets indeed may be modelled by this type of stochastic process. This is now regarded as a classical assumption. Many authors have attempted to introduce other processes more suitable to some special market conditions - e.g. markets with economic shocks (see e.g. [10]).

There are many approaches for implementing geometrical Wiener process for modelling movements of a given basic instrument. These include the mathematical theory of martingales, a limit transition for binary trees, or some other methods (see e.g. [18], [22]).

\subsubsection{Method of Iterative Stochastic Equation}

In this paper we will follow an approach proposed in [30] as most suited for our needs. Geometrical Wiener process and many other important stochastic processes for modelling interest movements of the underlying asset are special cases of what are we know as Levy processes. In this paper we present an approach suitable for these general processes.

The methodology used in the following can be called the Iterative Stochastic Equation method (in abbreviation ISE). It uses the concept of local characterizations for the Levy processes (see [34]). Let us now introduce the necessary concepts and basic facts, which can be also found in [30] and [34].

Let $\left(\Omega, \mathcal{F}, \mathcal{F}_{t}, \mathcal{P}\right)$ be a probabilistic space with right-continuous, complete filtration $\left(\mathcal{F}_{t}\right)_{t \in[0, T]}$, where $T<\infty$. The assumption that $T$ is finite is fulfilled in this paper because the financial instruments considered here have only finite life-time intervals.

Definition 1. A stochastic process $\left(Y_{t}\right)_{t \in[0, T]}$ is called a Levy process if it fulfills the following conditions:

1. $Y_{0}=0$ a.s. (almost sure),

2. $\left(Y_{t}\right)_{t \in[0, T]}$ has independent increments, 
3. for all $s \geq 0$ and $t \geq 0$ the random variable $Y_{t+s}-Y_{t}$ has the same distribution as $Y_{s}-Y_{0}$,

4. for almost all $\omega \in \Omega$ the trajectories of $\left(Y_{t}\right)_{t \in[0, T]}$ are right-continuous and have left-side limits - they are so called cadlag functions.

We assume that $\left(Y_{t}\right)_{t \in[0, T]}$ is an $\mathcal{F}_{t}$-adapted process. This means that for each time moment $\tau$ we know the whole behaviour of process $Y_{t}$ till $\tau$, i.e. the trajectory of $\left(Y_{t}\right)_{t \in[0, \tau]}$.

For Levy processes the local characterizations (i.e. the Jacod - Grigelionis characterizations) are the following functions:

$$
\begin{gathered}
B_{t}:[0, T] \rightarrow \mathbb{R}, B_{t}=b t, \\
C_{t}:[0, T] \rightarrow \mathbb{R}, C_{t}=c t \\
\nu:[0, T] \rightarrow \mathcal{M}(\mathbb{R}), \nu_{t}=\nu(d x) t, \nu(\{0\})=0, \quad \int_{\mathbb{R}}\left(|x|^{2} \wedge 1\right) \nu(d x)<\infty,
\end{gathered}
$$

where $b$ and $c$ are some constants, and $\mathcal{M}(\mathbb{R})$ is the space of measures on $\mathbb{R}$.

Let $\left(Y_{t}\right)_{t \in[0, T]}$ be the Levy process.

Definition 2. The following transformation of probabilistic measure for each $a \in \mathbb{R}$

$$
d P(a)=\exp \left(a Y_{T}-T\left(a b+\frac{a^{2} c}{2}+\int_{\mathbb{R}}\left(e^{a x}-1-a g(x)\right) \nu(d x)\right)\right) d P,
$$

where $g(x)=x \mathbb{1}(|x| \leq r)$ and $r$ is some constant connected with characterizations, is called the Esscher transformation.

Theorem 1. The parameters $(b(a), c(a), \nu(a))$ describing local characterizations of process $\left(Y_{t}\right)_{t \in[0, T]}$ in accordance with measure $P(a)$ are characterized by the following equations:

$$
\begin{aligned}
b(a) & =b+a c+\int_{\mathbb{R}} g(x)\left(e^{a x}-1\right) \nu(d x) \\
c(a) & =c \\
\nu(a)(d x) & =e^{a x} \nu(d x)
\end{aligned}
$$

In the following, we will be interested in the stochastic processes of the form

$$
\mathcal{S}_{t}=\exp \left(Y_{t}\right)
$$

where $\mathcal{S}_{t}$ is the transformation of process $Y_{t}$ to its exponential form. For example, if $Y_{t}$ is the arithmetical Brownian motion, then $\mathcal{S}_{t}$ will be geometrical Brownian motion.

Theorem 2. If the constant a satisfies the following conditions

$$
\begin{gathered}
b+\left(a+\frac{1}{2}\right)+\int_{\mathbb{R}}\left(e^{a x}\left(e^{x}-1\right)-g(x)\right) \nu(d x)=0 \\
\int_{\mathbb{R}}\left|e^{a x}\left(e^{x}-1\right)-g(x)\right| \nu(d x)<\infty
\end{gathered}
$$

then $\left(\mathcal{S}_{t}\right)_{t \in[0, T]}$ is a martingale in accordance to the measure $P(a)$. 
The proofs of above theorems can be found in [34].

Now we can use above theorems and definitions for our main aim. We are to acquire the iterative stochastic equation for stochastic process of interest rates movements of the underlying asset.

Let $r$ denotes a constant free-risk rate and

$$
\mathcal{Z}_{t}=e^{-r t} \mathcal{S}_{t}
$$

be the discounted process of the rates movements of the underlying asset. We have to find the formula for measure $Q$ equivalent to $P$ for which $\mathcal{Z}_{t}$ is a martingale. To solve this problem one should use definitions 1 and 2 and theorems 1 and 2 (for further details see [30] and [34]). The next step is to find a form of the process $\mathcal{S}_{t}$ according to the new probabilistic measure $Q$.

\subsubsection{Geometrical Wiener process}

Let us now illustrate the approach presented in Section 3.1.1 for the classical example of geometric Brownian motion.

We assume that the market operates in a continuous way and there are no additional transaction expenses and taxes. Let $\mathcal{W}_{t}$ denotes the standard (arithmetical) Brownian motion. Let $\sigma>0$ and $\mu>0$ be constants which we will call the volatility and drift respectively.

For the Black-Scholes model we have $Y_{t}=\mu t+\sigma \mathcal{W}_{t}$. Let $\mathcal{S}_{t}$ have the same meaning as in (7), i.e.

$$
\mathcal{S}_{t}=\mathcal{S}_{0} \exp \left(\mu t+\sigma \mathcal{W}_{t}\right)
$$

In this case the form of $\mathcal{S}_{t}$ for the equivalent measure $Q$ is

$$
\mathcal{S}_{t}=\mathcal{S}_{0} \exp \left(\left(r-\frac{1}{2} \sigma^{2}\right) t+\sigma \mathcal{W}_{t}^{Q}\right)
$$

where $\mathcal{W}_{t}^{Q}$ denotes the arithmetical Brownian motion for the measure $Q$.

For using Monte Carlo methods (see Section 3.2) we should change equation (12) to another form - the iterative stochastic equation (ISE). Let $[0, T]$ denotes the life time interval for the given financial instrument. We have to discretize $[0, T]$ into the set of time moments $\mathcal{T}=\left\{t_{0}=0, t_{1}, \ldots, t_{n}=T\right\}$, where $n$ is number of steps. We assume that distances between points in the set $\mathcal{T}$ are constant, i.e. $t_{i+1}-t_{i}=\Delta t=$ const for $i=1, \ldots, n-1$.

From the above discretization, the equation (12) changes to the form

$$
\mathcal{S}_{i+1}=\mathcal{S}_{i} \exp \left(\left(r-\frac{1}{2} \sigma^{2}\right) \Delta t+\sigma \sqrt{\Delta t} \epsilon_{i}\right),
$$

where $\epsilon_{0}, \epsilon_{1}, \ldots, \epsilon_{n-1}$ are iid (i.e. independent, identically distributed) random variables from $N(0,1)$ distribution. This sequential form, like (13), of the equations we call the iterative stochastic equations. The formula (13) is called an Euler scheme in the literature.

An example of geometrical Brownian motion trajectories generated from (13) is shown in Fig. 1.

It is generally known that if $\Delta t$ goes to 0 , the approximation given by ISE converges to the underlying originated stochastic process (see e.g. [34]). 
Value of underlying asset

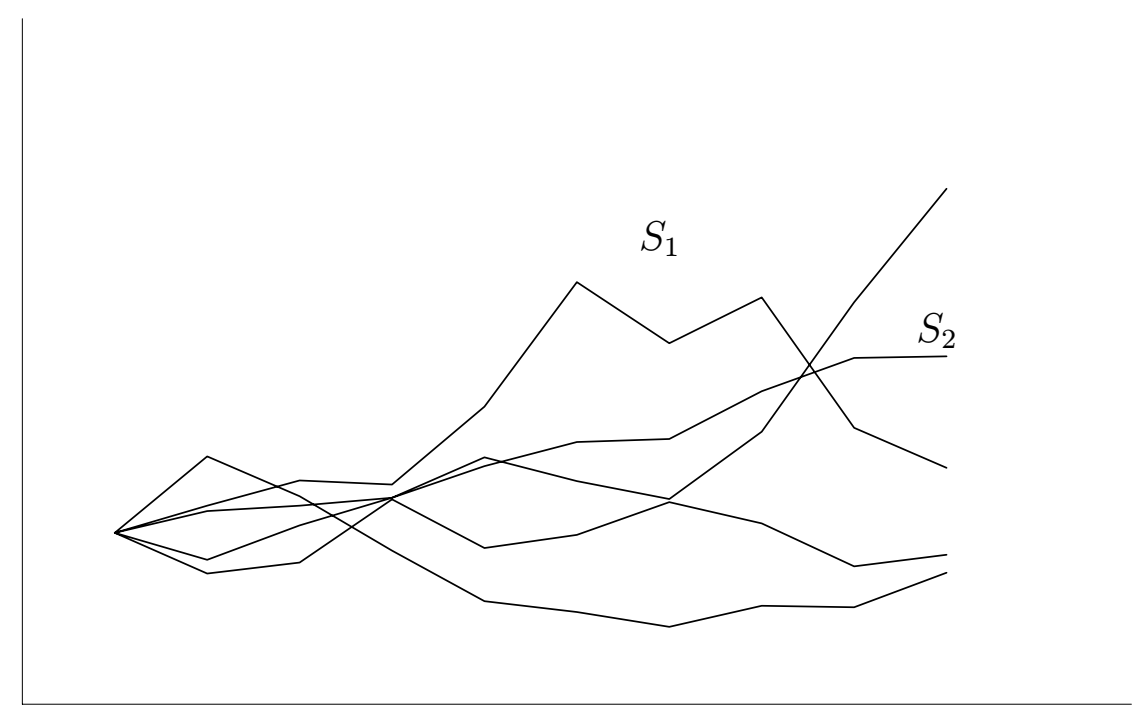

Time

Figure 1: Example of trajectories of geometric Brownian motion generated from ISE

\subsubsection{Other types of stochastic processes}

The method presented in Section 3.1.1 and 3.1.2 may be used for other types of the stochastic processes. Due to immense flexibility of Monte Carlo methods (see Section 3.2) we can use almost any kind of Levy process for modelling rates movements (see Section 4). This flexibility is easily seen in contrast to other methods. For example, binary trees method can not be used for stochastic processes with additional shocks, like Poisson jumps. Additionally, the presented method may be easily generalized for multidimensional stochastic processes.

There are only two serious limitations. The first one is the problem of estimating additional parameters of the given process. In the case of geometrical Brownian motion (Section 3.1.2) there are only two parameters - the volatility $(\sigma)$ and the risk-free rate $(r)$. The third parameter - drift $(\mu)$ - does not appear in the appropriate equation (12). But for more complex stochastic processes there may be additional necessary parameters.

The second problem arises from a necessity of solving some additional equations. These deterministic equations connect all parameters of the given process. To illustrate this let us consider an example from [30]. Assume

$$
Y_{t}=\mu t+\sigma \mathcal{W}_{t}+k\left(\mathcal{N}_{t}^{\kappa}-\kappa t\right)
$$

where $\mu, \sigma$ and $k$ are some constants, $\mathcal{W}_{t}$ is arithmetical Brownian motion and $\mathcal{N}_{t}^{\kappa}$ is a Poisson process with intensity $\kappa$ (hence $\kappa>0$ ). Processes $\mathcal{W}_{t}$ and $\mathcal{N}_{t}^{\kappa}$ are independent of each other.

From (8) we have

$$
\mu-k \kappa-r+\frac{\sigma^{2}}{2}+a \sigma^{2}+\kappa e^{a k}\left(e^{k}-1\right)=0 .
$$


This equation connects all parameters of the stochastic process of the form (14) and has to be solved with respect to variable $a$.

After changing the measure according to method presented in Section 3.1.1 we have

$$
\mathcal{S}_{t}=\mathcal{S}_{0} \exp \left(\left(\mu-k \kappa+a \sigma^{2}\right) t+\sigma \mathcal{W}_{t}^{Q}+\mathcal{N}_{t}^{Q \kappa \exp (a k)}\right)
$$

where $\mathcal{W}_{t}^{Q}$ is the Brownian motion with respect to the new measure $Q$ and $\mathcal{N}_{t}^{Q \kappa \exp (a k)}$ is the Poisson process with respect to $Q$ with intensity $\kappa e^{a k}$. We can now transform the equation (16) to the ISE form:

$$
\mathcal{S}_{i+1}=\mathcal{S}_{i} \exp \left(\left(\mu-k \kappa+a \sigma^{2}\right) \Delta t+\sigma \sqrt{\Delta t} \epsilon_{i}+k \rho_{i}\right),
$$

where $\rho_{0}, \ldots, \rho_{n-1}$ are iid random variables from Poisson distribution with intensity $\kappa e^{a k} \Delta t$. Other indicators have the same meaning as previous or in equation (13), respectively.

In order to apply equation (17), one should solve equation (15). This can be done by using numerical methods, e.g. the Newton method.

\subsection{Monte Carlo methods for pricing financial instruments}

We now introduce some basic concepts of Monte Carlo (abbreviated MC) methods for pricing financial instruments. The main idea is very simple and very flexible. It may be used for various processes of movements of the underlying asset and many types of financial instruments (see Section 4 and 5).

Let us assume that for a given financial instrument we know its life time interval $[0, T]$ and the initial value $\mathcal{S}_{0}$ of the underlying asset. This time interval is divided into $n$ steps (see Section 3.1.2). Starting with the value $\mathcal{S}_{0}$, from the appropriate ISE formula (e.g. (13) or (17)) we obtain values $\mathcal{S}_{1}, \ldots, \mathcal{S}_{T}$. These quantities are values of the basic instrument at time moments $t_{1}, \ldots, t_{n}=T$, respectively. They form the sample path $S^{1}$ of the stochastic process modelling the underlying asset movements.

The next step is the calculation of a payment of the given financial instrument for trajectory $S^{1}$. This payment we denote by $f\left(S^{1}\right)$. For example, for a European-style call option the payment is the function

$$
f\left(S^{1}\right)=\left(\mathcal{S}_{T}^{1}-K\right)_{+},
$$

where $\mathcal{S}_{i}^{j}$ is the value of the $j$-th sample path at time moment $t_{i}$ and $K$ is so called striking price (for additional information about options see [18]). In the following, we denote the general payment function by $f\left(\mathcal{S}_{t}\right)$.

In the same manner we can simulate $m$ trajectories $S^{1}, S^{2}, \ldots S^{m}$ of the desired stochastic process $\mathcal{S}_{t}$. We can also calculate $m$ payments $f\left(S^{1}\right)$, $f\left(S^{2}\right), \ldots, f\left(S^{m}\right)$ for the given financial instrument from the simulated sample paths, where $m$ is the simulations number.

The last step is a calculation of a discounted average of the payments sequence:

$$
\mathcal{C}^{m}=e^{-r T} \frac{1}{m} \sum_{i=1}^{m} f\left(S^{i}\right),
$$


where $r$ is the risk-free rate (see Section 3.1.1).

Being more careful, we should write the formula (19) in another form:

$$
\mathcal{C}^{m}=e^{-r T} \frac{1}{m} \sum_{i=1}^{m} \mathrm{FV}_{T} f\left(S^{i}\right),
$$

where $\mathrm{FV}_{T}(\mathcal{Y})$ is the future value of cash flow $\mathcal{Y}$ at time moment $T$.

Formula (20) is needed in cases when payments are realized at various time moments, not only at the end of time interval. In such cases there is a need to transform all these payments to the same time moment $T$ in order to calculate correct present value. However, in the following we refer to the formula (19) keeping in mind the more proper form (20).

It is easy to see that the sample paths $S^{1}, \ldots S^{m}$ form an iid sequence. Then, from the Strong Law of Large Numbers (in abbreviation SLLN) we have

$$
\mathcal{C}^{m} \underset{m \rightarrow \infty}{\stackrel{a . s .}{\longrightarrow}} C
$$

where $C$ denotes the real price for the given financial instrument - the present value of this instrument.

There are some necessary assumptions for using SLLN and therefore formula (21). We assume that both average and variance of $f\left(\mathcal{S}_{t}\right)$ are finite, i.e. $\mathbb{E} f\left(\mathcal{S}_{t}\right)<\infty$ and $\operatorname{Var} f\left(\mathcal{S}_{t}\right)<\infty$.

\subsubsection{Antithetic variables method}

As it was mentioned before, the sample paths $S^{1}, \ldots, S^{m}$ form the iid sequence. In accordance with this property we may easily estimate variance of the average $\mathcal{C}^{m}$ and calculate appropriate confidence intervals by using the Central Limit Theorem.

It is generally known that convergence of $\mathcal{C}^{m}$ to its real value $C$ is of order $\frac{\bar{\sigma}}{\sqrt{m}}$, where $\overline{\sigma^{2}}$ is the estimator of the variance. To reduce necessary amount of simulations one should minimize the value of this variance. This issue is very important. Increasing the simulations number by factor 100 gives the reduction of the confidence intervals only by factor 10 . And decreasing standard deviation by factor 100 gives the reduction by the same factor.

There exists different types of variance reduction techniques. We focus in this paper only on one of them. The examples of other methods are fast Monte Carlo, control varieties method, etc. (see e.g. [32]).

A relatively simple and reliable technique is the antithetic variables procedure. Let us use this method for the geometrical Brownian motion (see Section 3.1.2). In this case we should generate iid random variables $\epsilon_{0}, \ldots, \epsilon_{n-1}$ from $N(0,1)$ distribution only for odd sample paths $S^{1}, S^{3}, \ldots$ (see (13)). For the even sample paths we use random variables generated before. To explain this procedure let us introduce additional notation.

Let $\epsilon_{i}^{j}$ denote a random variable generated for $t_{i+1}$ time moment for $j$-th sample path. From the above assumption we have $\epsilon_{i}^{j}$ only for $i=0, \ldots, n-1$ and $j=$ $1,3,5, \ldots$ Let $\epsilon_{i}^{j+1}=-\epsilon_{i}^{j}$ for $i=0, \ldots, n-1$ and $j=1,3,5, \ldots$.

In Fig. 2 we can see two trajectories. The sample path on the right-hand side is constructed from the left-hand side trajectory after application of this method. 

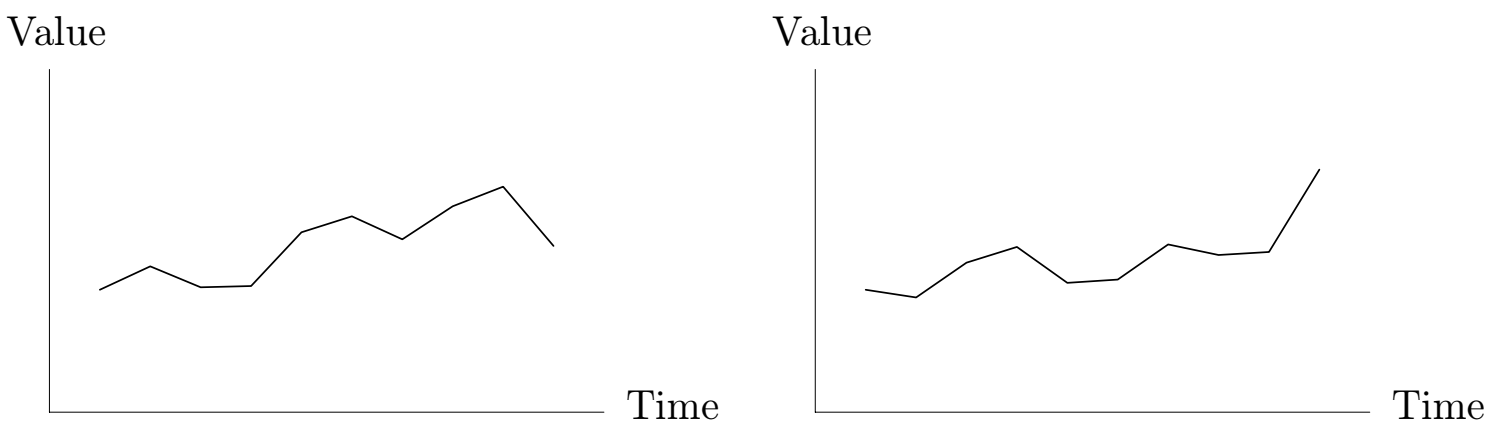

Figure 2: Example of applying antithetic variables method

Under such requirements it is easy to see that with this modification the equation (21) is still fulfilled. Moreover, the total variance of $\mathcal{C}^{m}$ is reduced, because

$$
\operatorname{Cov}\left(S^{j}, S^{j+1}\right)<0
$$

for $j=1,3,5, \ldots$.

It is easy to see that the antithetic variables method may be used for any stochastic process with the Brownian motion part - e.g. for the one presented in Section 3.1.3. This method is one of the simplest to use in computer algorithm and it is rather universal, because it is independent of the kind of the financial instrument.

\subsection{Catastrophe bonds}

As previously mentioned, catastrophe bonds (in abbreviation: cat bonds) are relatively new and fast developing financial instrument. They were introduced in 1995, but become wider known after 1996. In April 1997, USAA, an insurer from Texas, initiated two new classes of cat bonds: A-1 and A-2. They were worth approximately $\$ 447$ million (see [15], [17], [28]).

As it was emphasized in Section 1, there is one important difference between cat bonds and other financial instruments. The cat bond is always connected with occurrence of some natural catastrophe in specified region in fixed time interval, e.g. earthquake in California to the end of the year. The event, which is precisely described, is called the triggering point (see e.g. [15], [24], [29]).

For example, the A-1 USAA bond was connected with hurricane on the east coast of USA between July 15, 1997 and December 31, 1997. The payment from this bond depended on London InterBank Offering Rate (LIBOR) and equalled LIBOR plus 282 basis points (interest rate plus $2.82 \%$ ).

If there had been a hurricane in mentioned above region with more than 1 billion $\left(10^{9}\right)$ dollars loses against USAA, the coupon - the additional payment of this bond - would have been lost. The principal, the main payment of $\mathrm{A}-1$ bond, was always guaranteed (see [15], [17]). In case of A-1 bond the triggering point was cumulated loss amount from catastrophe (hurricane). As we can see from this example, the triggering point changes the structure of payment for the cat bond.

There are now other types of cat bonds. They may be related to other types of triggering points - e.g. to magnitude of earthquake, the losses from flood, etc. (see e.g. [23], [28], [35]). 
The main aim of cat bonds is to transfer risk from insurance markets and governmental budgets to financial markets.

Insurance markets in many countries have insufficient capacity and liquidity of risk reserves and resources. For example, between 1989 and 1995 total insured losses from natural catastrophes amounted to $\$ 75$ billion, $50 \%$ higher than in entire preceding 38-years period (see [15]). Base upon predictions of global climate change, the losses from natural catastrophes are predicted to be even higher in next years. Direct damages from natural disasters have increased dramatically (see [4]).

The insurance markets are relatively small in comparison with possible demands. For example, in the USA the capital of insurance market amounts to $\$ 245$ billion but the property in this country is worth approximately $\$ 25-\$ 30$ trillion $\left(10^{12}\right)$. A tremendous catastrophe could destroy insurance reserves and drive many insurance companies into bankruptcy, even in wealthy and highly industrialized country. More than $20 \%$ of insurance capital may be lost in one serious catastrophe (see [17]). The lack of capacity and liquidity of insurance markets are also discussed in [6], [12], [15], [20].

The situation is even worse in developing countries. In such countries governments often have very tight budgets and can not reserve sufficient money for infrequent but also major catastrophes (see [3], [29]). In cases of such catastrophes they have to use foreign help, thus increasing their debt. This may has a very serious negative effects on the national economy (see e.g. [3], [25]). Huge natural catastrophes have long-term negative effects and increase poverty of people in developing countries (see e.g. [3], [8], [13], [25]).

In contrast to insurance markets, financial markets have enough resources and liquidity of funds. Their capital is estimated to be $\$ 19$ trillion. Additionally, even enormous change in resources amount is not a problem for financial markets, because their approximate daily standard deviation equals to $\$ 133$ billion (see e.g. [15]). Such a large change in capital, routine for financial markets, would be destructive for insurers and very troublesome for almost all governments. In this light it is easily seen that there are advantages in transferring catastrophe losses to financial markets by using risk-transfer financial instruments, e.g. by cat bonds.

One of the most important questions related to cat bonds is the problem of pricing - i.e. how much should we pay for a given cat bond after fixing its structure of payments? In the language of economics, this issue is the problem of calculating the present value of cat bond after establishing the types and amount of payments in accordance with triggering point.

This question is especially very significant in risk management.

Answers for this question is important for providers of cat bonds. For example, to establish a new type of cat bond - like FloodBond (see [23]), the seller (e.g., the Polish government), has to find the present value for this new instrument. Without this knowledge, further steps in selling this cat bond are impossible. For further discussion see Section 6.

In Section 4 we use Monte Carlo and ISE methods to present general outline for pricing financial instruments and application to cat bonds (see Section 4.1). After these, we show examples of using these methods (see Section 5). 


\subsection{EDGE software}

The software package EDGE, an Earthquake and Damage Generator / Estimator for Toscana, was developed at IIASA (see [1], [33]). This software consists of two main parts. The first part is a generator of catastrophes (earthquakes), and the second is an estimator of damages arising from generated catastrophes.

The generator of catastrophes simulates earthquakes. From input calibrated to specified region it generates scenarios of possible earthquakes for this region. These scenarios include a variety of important data, i.e. locations of earthquake occurrences, their magnitudes, affected areas, etc. (see [1]).

These scenarios are then used in the second part of the software. Using additional input, the estimator creates distributions of possible losses and samples of losses for different locations in the whole region. These outputs may be used, e.g., by insurers or more generally by risk managers to design loss-reduction or loss-spreading programs (see e.g. [1], [8], [25]).

But, as we will see, EDGE software may be used in many other ways. In particular, we use procedures originated from EDGE in our examples (see Section 5) for a problem of catastrophe bond pricing.

We should keep in mind that EDGE software, despite its generality, is always calibrated for the given region. It may be used for different regions, but it requires the region specific initial data. Inputs consist of statistical and geological data for the given region - maps of maximum observed macro-seismic intensities, maps of the geo-tectonic structure, etc. The initial data is used then in Monte Carlo algorithm embedded in the scenario generator.

Besides purely statistical data, the geopsychical models, e.g. the GutenbergRichter law, can be used as well to produce synthetic catalogues of earthquakes. The Gutenberg-Richter law, which connects intensity of an earthquake with its occurrence time, is also used in other numerical approaches (see e.g. [9], [11]).

For damage estimator there is a need of additional inputs on regional values of property and their vulnerability, e.g. the vulnerability of a given type of buildings against shaking intensities. In [1] EDGE is calibrated for Toscana region in Italy.

In general, simulations of earthquakes and losses scenarios are complex stochastic processes. For more technical details we refer the reader directly to [1].

However, it is worth mention that this software is very well suited for purpose of pricing cat bonds via combination of ISE and Monte Carlo method. Additionally both EDGE software and cat bonds are designed for specific region.

We should also emphasize that similar software was also designed for flood simulations (see [8]), which may also be utilized for pricing catastrophe bonds (see Section 6).

\section{General outline}

After introducing basic foundations and notations in Section 3 we may now present a general model. This generalization will be used in Section 5 for pricing in three examples of catastrophe bonds (see Section 3.3).

The general model of the pricing of any given financial instrument consists of 
four steps. In the first step one should choose an adequate type of stochastic process for modelling movements of the underlying asset. These underlying assets may be indexes, prices of stocks, exchange rates of banks and many other rates and prices.

From mathematical point of view this stochastic process may be one of the Levy processes. This, in particular, opens up the possibility of using martingale theory, which provides a powerful tool for handling problems of financial mathematics (see e.g. [34]). Of course there is possibility to use other types of processes. However this is hardly ever found in financial literature. For a family of the Levy processes, suitable theorems and definitions are presented in Section 3.1.

From a practical point of view the chosen stochastic process should be suitable for behaviour of the given market (for further discussion see e.g. [7]). For example the geometrical Brownian motion (see Section 3.1.2) may be very useful to model a market index or price of share. But other kinds of processes may be chosen without creating additional problems.

The next step is the simulation of the desired stochastic process. In order to do this, one should apply appropriate ISE (iterative stochastic equation). Examples of these equations for two Levy processes are presented in Section 3.1.2 and 3.1.3. The very significant issue is the number of steps $n$ and number of simulations $m$. Nowadays, in the age of fast and relatively cheap computer resources one may be tempted to determine a great amount of simulations without any accelerating procedure. However, in practice it is often important to apply some accelerating methods, like the antithetic variables procedure (see Section 3.1.3) or quasi Monte Carlo (see e.g. [14]), especially when we are concerned with the designing of multidimensional optimal portfolios of cat bonds (see e.g. [5]).

The third step is calculation of the payments of the given financial instrument for all sample paths simulated before.

This procedure strictly depends on the nature of the instrument, i.e. on a plan of payments for this instrument which is put down in its lawful description. As we will see in our examples (see Section 5) this step may be quite complex and require additional simulations and extra embedded software.

The last step is calculation of the discounted average of the payments according to equation (19). This average is called the price (present value) of the given financial instrument.

Of course there is a possibility that this price may be different from the real price in the absence of the arbitrage-free market. Still we do not deal with this issue, because the method presented in this paper may be treated as a first preliminary step for the seller of a new cat bond (see Section 6).

Other additional necessary conditions for the market are specified in Section 3.1.1 and Section 3.1.2.

We should once more emphasize that this four-step algorithm is very flexible and general. It may be used for many types of underlying stochastic processes and various kinds of financial instruments (options, bonds and so on).

\subsection{Application to cat bonds pricing}

We apply the general method presented in Section 4 to problem of cat bonds pricing. In case of the cat bond for each trajectory $S^{i}$, which is realization of process 
$\mathcal{S}_{t}$, there is also a scenario of catastrophe $X^{i}$. This scenario is generated by some stochastic process $\mathcal{X}_{t}$ or random variable $\mathcal{X}$. In the following we denote it simply $\mathcal{X}$ when no confusion can arise.

Trajectories of underlying asset movements and scenarios of catastrophe form pairs $\left(S^{i}, X^{i}\right)$, where $i=1, \ldots, m$. These pairs are used for generating payments. The payment function in case of cat bonds has the form $f\left(\mathcal{S}_{t}, \mathcal{X}\right)$, i.e. it depends on both processes. However, this is only a slightly difference from related equations of Section 3.2. The formula

$$
\mathcal{C}^{m}=e^{-r T} \frac{1}{m} \sum_{i=1}^{m} f\left(S^{i}, X^{i}\right)
$$

is an equivalent of (19). And the equation

$$
\mathcal{C}^{m}=e^{-r T} \frac{1}{m} \sum_{i=1}^{m} \mathrm{FV}_{T} f\left(S^{i}, X^{i}\right)
$$

is an equivalent of (20).

Of course the convergence (21) is fulfilled if

$$
\begin{gathered}
\mathbb{E} f(\mathcal{S}, \mathcal{X})<\infty \\
\operatorname{Var} f(\mathcal{S}, \mathcal{X})<\infty
\end{gathered}
$$

In the following we always assume that these conditions are satisfied.

\section{$5 \quad$ Examples}

Let us now apply formulas (23) - (24) for some examples.

\subsection{Introductory example}

Background First, we begin with the same example as in Section 2. As we have seen, for this example there is no necessity to apply Monte Carlo methods. Thus this example may serve as a good test problem and as an introduction for next two more complex examples.

As previous, suppose that the geometrical Brownian motion (see Section 3.1.2) models the movements of the underlying asset. This process is described by two parameters: volatility $\sigma$ and risk-free rate $r$.

If by the time $T$ there is a catastrophe, the purchaser of the bond will receive no payment. Otherwise, he will receive a payment of the quantity $\mathcal{S}_{T}$ at time moment $T$, i.e. the full price of index in time $T$. Besides, there are no other payments from this instrument.

The time of catastrophe occurrence is a random variable $\mathcal{X}$, exponentially distributed with intensity $\lambda$ (see (1)). The stochastic process of movements of index $\mathcal{S}_{t}$ and time of catastrophe $\mathcal{X}$ are independent variables. 
Algorithm In this example the payment function is given by (3).

Let GenPoiss denote the procedure of generating random variable from Poisson distribution with intensity $\lambda$ and GenNorm similar generating algorithm for $N(0,1)$ distribution. Set $n$ for the number of steps and $m$ for the number of simulations.

With this notation we have the following algorithm in pseudocode for this example:

Algorithm I

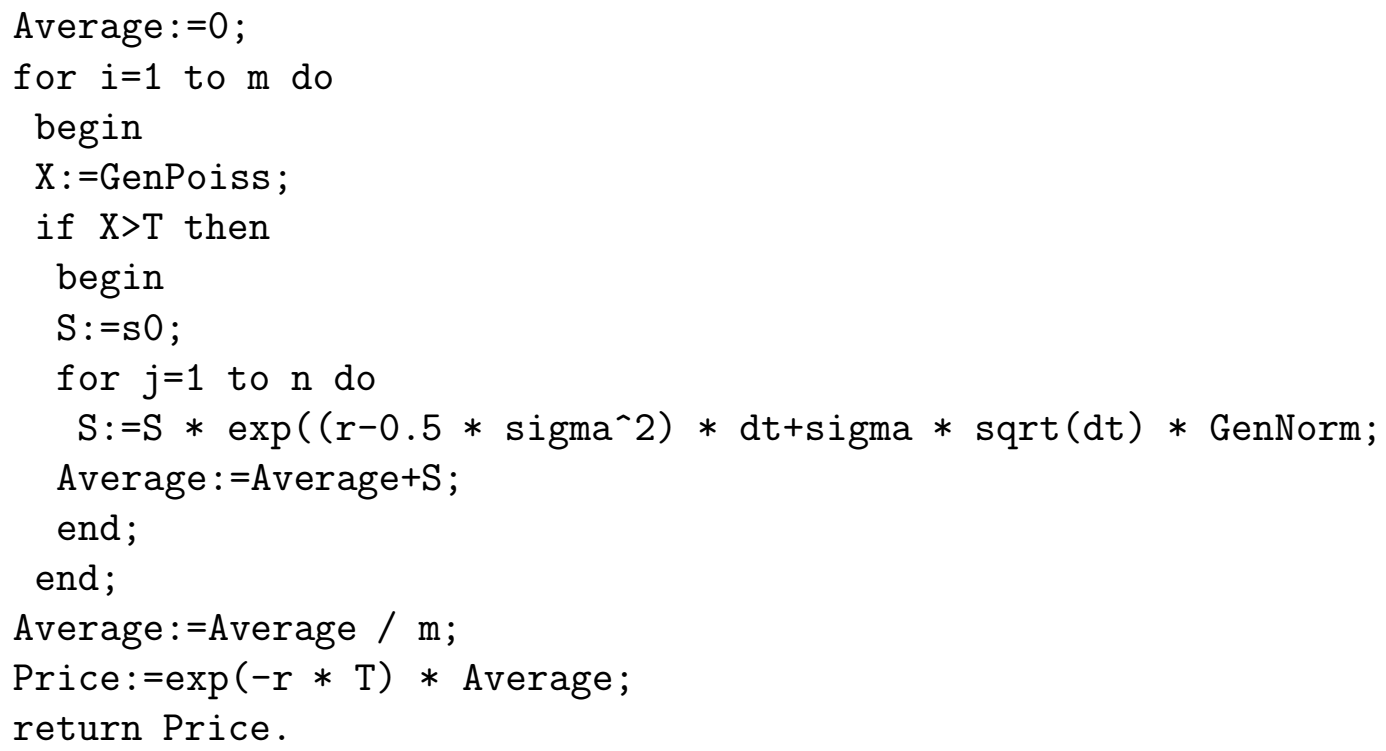

where Price is the price (the present value) of the cat bond, s0 is the starting value of index $\left(\mathcal{S}_{0}\right)$, dt is the time interval between steps $(\Delta t)$ and sigma is the volatility of the index $(\sigma)$. Other notations have the same meaning as previous.

In the light of Section 4.1 this algorithm is easy to understand and there is no need for additional explanations. The example of pairs $\left(S^{i}, X^{i}\right)$ generated by the algorithm may be found in Fig. 3 .

Application We begin with some very general remarks which are also useful for the next both examples.

In our algorithm two special procedures are used: GenPoiss and GenNorm. These procedures generate random variables from appropriate distributions. In practical applications it is very important that they should be fast and statistically reliable. We now look on these requirements in more detailed way.

It is generally known that for all types of distributions the uniform (pseudo)random number generator is necessary. This generator has to be fast and statistically stable, i.e. it has to pass some statical tests. A good example of such a generator is Mersenne Twister (see e.g. [26]). Of course, there is also a need for good procedures to transform uniform random variable to variable from required distribution. Such a procedure also has to satisfy both of mentioned above requirements. For example to apply the algorithm of transforming uniform random variable to one from normal distribution an inverse Moro distribution may be very useful (see [27]).

Additionally, to improve the speed of the algorithm the antithetic variables method (see Section 3.2.1) or other types of accelerating methods may be easily used. 


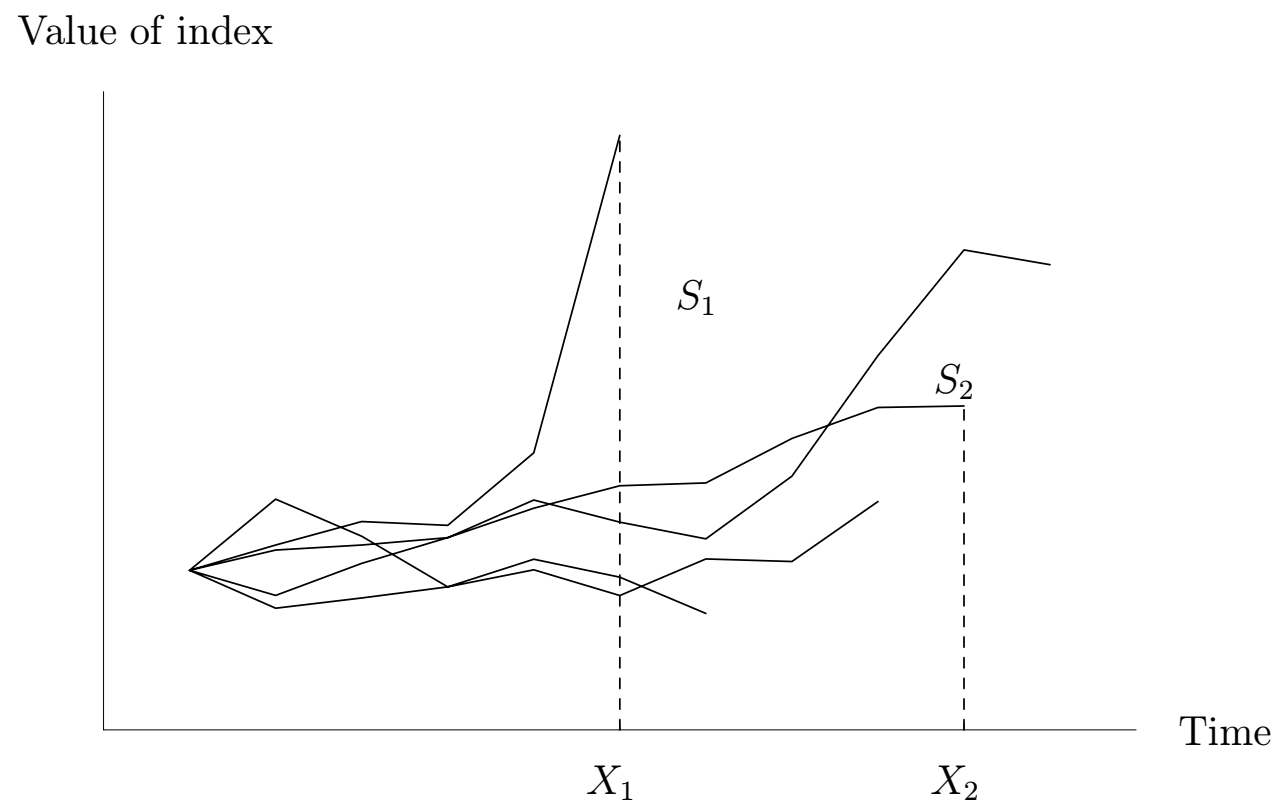

Figure 3: Example of pairs of index movements trajectories and catastrophe scenarios

\subsection{Cat bonds depending on magnitude}

Background The next example describes the catastrophe bonds with payments depending on a magnitude of an earthquake (in abbreviation CBDME). In the algorithm we will use procedures from EDGE software.

Suppose that our financial instrument, which is called CBDME, satisfies the following conditions:

1. Payments are modelled by movements of some basic instrument. The process of these movements $\mathcal{S}_{t}$ is the geometrical Wiener process with the volatility $\sigma$ and the risk-free rate $r$.

2. Both parameters $r$ and $\sigma$ are constant in time.

3. Additional requirements concerning the market from Section 3.1.1, 3.1.2 and 4 are fulfilled.

4. CBDME is written for the region for which necessary input to EDGE software is available (see Section 3.4).

5. CBDME has the life time interval $[0, T]$, where $T=k l$ for some $k, l \in \mathbb{N}$.

6. The structure of the payments of CBDME is the following: if till time $p l$, where $p \leq k$, there is no catastrophe of magnitude above $R$ degrees in the Richter's scale, then the purchaser of CBDME will receive the payment $\mathcal{S}_{p l}$, 
where $\mathcal{S}_{p l}$ is the full price of the underlying asset at time moment $p l$. If till time $p l$ there is catastrophe of magnitude above $R$, then the purchaser will not receive any payments after the moment of catastrophe.

7. If $\mathcal{X}$ denotes the random variable of time moment of earthquake above $R$ degrees, the process $\mathcal{S}_{t}$ and $\mathcal{X}$ are independent of each other.

In this example the life time interval $[0, T]$ of a cat bond is divided into $k$ subintervals. For example, the bond may be prescribed for 5 years, i.e. 60 months $(T=60)$ and this five-years interval may be divided into half-years (hence $l=6$ and $k=10$ ). Of course, unit of time measure - a month in the above example - depends on practical application.

The purchaser will receive payment after each half-years if there is no catastrophe. The amount of this payment depends on current rates in the end of the given subinterval. If for example no earthquake occurs during five years, he will get a sequence of payments $\mathcal{S}_{6}, \mathcal{S}_{12}, \ldots, \mathcal{S}_{60}$ - ten payouts in general. And if there is a triggering point at some time moment $X<T$, where $X$ is the realization of random variable $\mathcal{X}$, the purchaser will obtain no payments after this moment. For example - if in the second half of the third year there is an earthquake above $R$ degrees, the buyer will receive only five payments: $\mathcal{S}_{6}, \mathcal{S}_{12}, \ldots, \mathcal{S}_{30}$.

The similar kinds of cat bonds as described above are used in practice (see Section $3.3)$.

Algorithm Now we can transform above specification into an algorithm.

In this algorithm we use a special procedure from EDGE software. This procedure is called GenEarthquakeTime $(R)$. The input to this procedure is the same as described in Section 3.4 for EDGE. Moreover, the additional parameter $\mathrm{R}$ is required. This parameter describes maximal magnitude of earthquake $R$ for which there are still payments for purchaser of CBDME (see point 6 in previous subsection). The output from GenEarthquakeTime ( $\mathrm{R}$ ) is the time moment $X$ of the triggering point, i.e. the earthquake of magnitude above $R$ degrees.

In accordance with previous paragraph, the payment function $f\left(\mathcal{S}_{t}, \mathcal{X}\right)$ or, more precisely, future value of cash flow from sample path $S$ and for time moment $X$ is described by

$$
\mathrm{FV}_{T} f(S, X)=\mathrm{FV}_{T}\left(\mathcal{S}_{l}, \mathcal{S}_{2 l}, \ldots, \mathcal{S}_{\tau l}\right)=\sum_{d=1}^{\tau} \mathrm{FV}_{T}\left(\mathcal{S}_{d l}\right)
$$

(see (24) and appropriate remark in Section 3.2), where

$$
\tau=\min \left\{k,\left[\frac{X}{l}\right]\right\}
$$

and $[z]$ denotes the absolute value of $z$.

To simplify the algorithm suppose that we have procedure Tau (X) which calculate expression (28).

As in the previous example, set $n$ for the number of steps and $m$ for the number of simulations. For simplicity of algorithm let us assume that we can write parameter $n$ in the form $n=k l s$ for some $s \in \mathbb{Q}_{+}$where $l s \in \mathbb{N}$. 
Under such assumptions we can write the following algorithm

Algorithm II

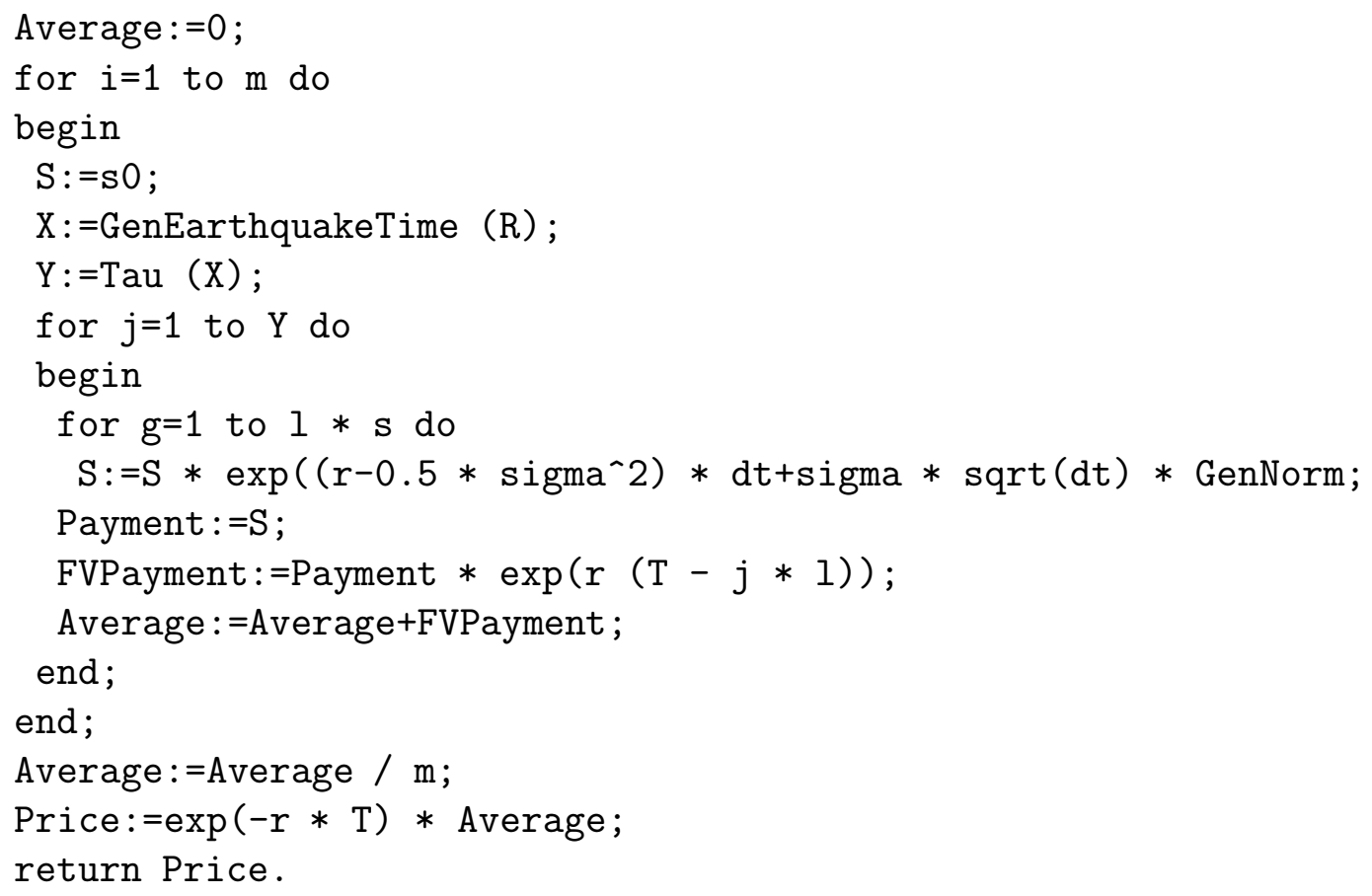

This algorithm is more complex than in the introductory example, so we should describe it in more detailed way.

There are three loops in the algorithm. The external loop with a counter $i$ is used for running $m$ simulations. After this loop the average (variable Average) from these simulations is calculated and the price (variable Price) according to (23) is computed as well.

For each simulation the time $\mathrm{X}$ to the nearest earthquake of magnitude above $R$ degrees is calculated. The procedure Tau (X) transforms this time to the number $Y$ of full subintervals with payments for purchaser of CBDME.

The quantity $\mathrm{Y}$ is used as the upper bound for the second loop with a counter $j$. In this loop the payment after each of subintervals is calculated (variable Payment). Next, the future value of this payment is computed with free-risk rate $r$ for the time moment $T$ (variable FVPayment). This value is then added to other payments stored temporarily in variable Average.

The internal loop with counter $\mathrm{g}$ is used for simulating the sample path of the underlying asset for one subinterval (variable $\mathbf{S}$ ). After the end of the subinterval the value of this trajectory is the input for the payment calculation.

It is very important to notice that for each of payments we compute their $f u$ ture values and then discount them, because the payments take places in different moments of time. So they have to be "transferred" to the same moment $T$ before discounting (see appropriate remark in Section 3.2 and (20), (27)).

Application Apart from general remarks like in Section 5.2, we can make some additional ones.

As it is easy to see, in the algorithm II we have to make $m$ simulations with $n$ steps for each simulation. These $n$ steps are divided into $k$ subintervals and $l s$ steps 
for one subinterval. In practical applications it is very important to decide how to locate computional time into the quantities $m$ and $n$.

One may be tempted to run many simulations with few steps, hence $m$ will be large and $l s$ very low - after fixing the type of bond one can not change the value $k$. Others may choose an inverse way. We advise to choose as many simulations as it is possible and reduce amount $l s$ even to 1 . Such an approach is dictated by two issues.

First, it is easy to see that in case of geometrical Brownian motion and for the payment at the end of the subinterval the generation $l s$ steps and simulation only one with appropriate change of time interval in (13) give the same results. Words "the same results" should be understood in their stochastic meaning, i.e. that processes simulated by taking $l s=1$ and $l s>1$ give stochastically the same payments. This follows from the form of finite-dimensional distributions of Wiener process and property 2 of the Levy processes (see Section 3.1 and [34]).

The second issue is connected with EDGE software. For each calling of the GenEarthquakeTime ( $R$ ) procedure there is a necessity to produce one scenario of an earthquake. The generation of such a scenario takes some computional time. And because earthquakes are events with small probability of occurrence, it seems that realization of many number of simulations is the right way to estimate price for the cat bond with relatively small error. So it seems that locating computional resources to gain more simulations is better way than achieving more steps in one simulation.

\subsection{Cat bonds depending on amount of losses}

Background In this section we deal with another, more complex example. In contrast with previous example, this cat bond depends on the cumulated level of losses caused by earthquakes. We use abbreviation CBDCLL for such a kind of financial instrument. As previous we use procedure from EDGE software.

Suppose that we have $\alpha$ layers of the loss levels. Each possible layer is described by lower and upper bounds $c_{i}$. These bounds present a given layer of losses, i.e. interval of cumulated losses. The upper bound of $i$-th layer is equal to the lower bound of $i+1$-th layer. So we have the sequence $\mathcal{C}=\left(c_{0}, c_{1}, \ldots, c_{\alpha}\right)$, where $c_{i}$ is the upper bound of $i$-th layer and $c_{0}$ is the lower bound of the first layer. Of course the natural condition

$$
0 \leq c_{0}<c_{1}<\cdots<c_{\alpha} \leq \infty
$$

has to be fulfilled.

As in previous example, set $[0, T]$ for the life time interval of CBDCLL and $k$ for the number of subintervals and $l$ for the length of subinterval.

Let $\mathcal{P}_{f}$ denote the $\alpha \times k$ matrix of real functions

$$
\mathcal{P}_{f}=\left(f_{i j}(\mathcal{S})\right)_{i=1, \ldots, \alpha ; j=1, \ldots, k},
$$

where entry $f_{i j}(S)$ is the payment for sample path $S$ after the $j$-th subinterval if the cumulated loss level to this moment reaches the $i$-th layer. Of course the function $f_{i j}(\mathcal{S})$ should be $\mathcal{F}_{j l}$-measurable, i.e. it has to depend only upon behaviour of $\mathcal{S}_{t}$ 
to $j$ subinterval inclusive, not on the future of this process. Assume additionally that each of these functions has no constant part, i.e. the part independent of the trajectory $S$.

Let $\mathcal{P}_{d}$ denote the $\alpha \times k$ matrix of constants

$$
\mathcal{P}_{d}=\left(d_{i j}\right)_{i=1, \ldots, \alpha ; j=1, \ldots, k},
$$

where entry $d_{i j}$ is the constant payment, independent of process $\mathcal{S}_{t}$, after the $j$-th subinterval if the cumulated loss level to this moment reaches the $i$-th layer.

With this notation we can describe our desired financial instrument. Suppose that CBDCLL satisfies the following conditions:

1. The same conditions as in points $1-5$ from Section 5.2 are also satisfied for CBDCLL.

2. The structure of the loss layers is given by the sequence $\mathcal{C}=\left(c_{0}, c_{1}, \ldots, c_{\alpha}\right)$.

3. The cumulated loss level from the earthquakes till time moment $t$ is modelled by a stochastic process $\mathcal{X}_{t}$.

4. The structure of the payments of CBDCLL is the following: if to the end of $j$ th subinterval $(j \leq k)$ the cumulated amount of losses is in layer $i(i \leq \alpha)$, i.e. $\mathcal{X}_{j l} \in\left[c_{i-1}, c_{i}\right]$, then purchaser will get payments $f_{i j}(\mathcal{S})$ and $d_{i j}$. The amount $f_{i j}(\mathcal{S})$ depends on the behaviour of the process $\mathcal{S}_{t}$ till the end of subinterval $j$, i.e. $\left(\mathcal{S}_{t}\right)_{t \in[0, j l]}$. The quantitative $c_{i j}$ is independent of this process.

5. Stochastic processes $\mathcal{S}_{t}$ and $\mathcal{X}_{t}$ are independent of each other.

The condition 5 is discussed in the subsection Application.

Now we can describe the payment schedule for CBDCLL in more detailed way. As we see from 4 , after each of subintervals $j$, where $j=1, \ldots, k$ the value $X_{j l}$ of the stochastic process $\mathcal{X}_{t}$ of cumulated amount of losses from earthquakes is computed. This quantity is a basis for calculating the currently payment for the purchaser. It decides which of the layer of losses is chosen. Hence, which entries from matrixes $\mathcal{P}_{f}$ and $\mathcal{P}_{d}$ are used for counting of the payment. If we have $i$-th layer of losses (i.e. $\left.X_{j l} \in\left[c_{i-1}, c_{i}\right]\right)$, the buyer at time moment $j l$ will receive the payment

$$
f_{i j}(S)+d_{i j}
$$

The part of payment $f_{i j}(\mathcal{S})$ depends on the movements of the underlying asset only till the end of subinterval $j$, i.e. the time moment $j l$. In more mathematical language it depends only upon behaviour of stochastic process $\left(\mathcal{S}_{t}\right)_{0 \leq t \leq j l}$.

Of course, the matrixes $\mathcal{P}_{f}$ and $\mathcal{P}_{d}$ of payments in real-life problems should be chosen in enough logical way, i.e. not leading to inconsistencies. However, we do not present special mathematical conditions for them here. 
Algorithm In the light of previous paragraph, we assume that we have three special procedures.

The first one comes from EDGE software and it will be called as GenCumLossAmount ( $W$ ). The input $W$ is the chosen time moment and the output is the cumulated amount of losses till $\mathrm{W}$.

The second procedure is GenPaymentF ( $\mathrm{X}$, Table, $\mathrm{W}, \mathrm{St}$ ). The inputs are: $\mathrm{X}$ - the cumulated amount of losses (the output from the previous procedure); Table - the name of the matrix; W - the time moment and St - the values of the process $\mathcal{S}_{t}$ till time $\mathrm{W}$, i.e. the behaviour of trajectory $S$ till this time moment. This procedure is used to generate a payment dependent on the process $\mathcal{S}_{t}$. The output is the appropriate entry, i.e. the suitable function $f_{i j}(\mathcal{S})$ of matrix Table (see (30)) for described above inputs $\mathrm{X}, \mathrm{W}$ and $\mathrm{S}$ (see point 4 in the previous subsection).

The third procedure, GenPaymentD (X, Table, W) is very similar to the last one. The inputs X, Table, $\mathrm{W}$ are the same as in GenPaymentF (X, Table, W, St). This procedure is used to generate a payment independent on the process $\mathcal{S}_{t}$. The output is the appropriate entry, i.e. the suitable constant $d_{i j}$ of matrix Table (see (31)) for above inputs $\mathrm{X}$ and $\mathrm{W}$ (see point 4 in the previous subsection).

Algorithm III

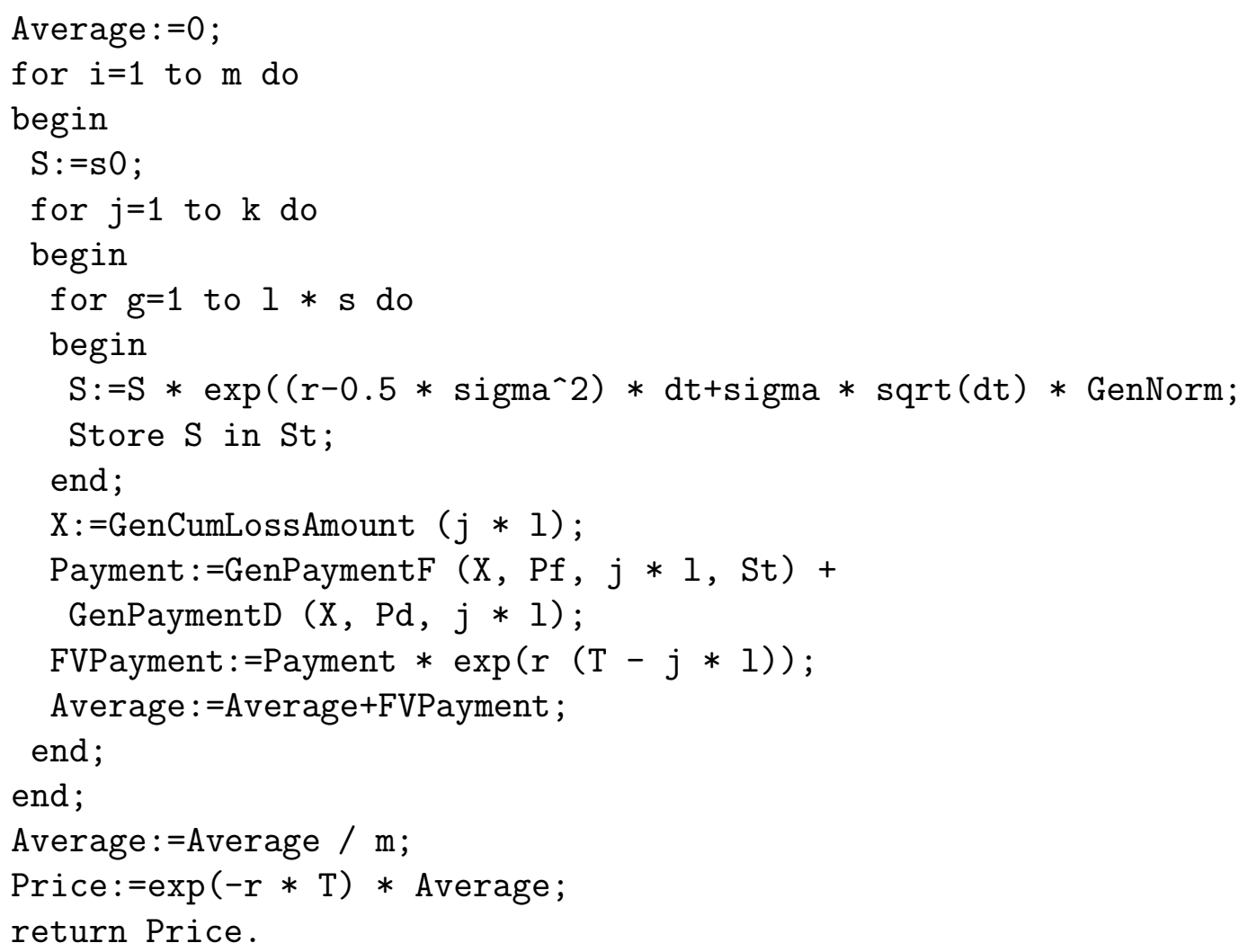

where Pf is the matrix $\mathcal{P}_{f}$ and Pd is the matrix $\mathcal{P}_{d}$ (see (30) and (31)).

As we can see, this algorithm is similar to the one presented in Section 5.2. Here we have also three loops. There are only three issues which we should explain in more detailed way.

The first one is the procedure Store. As it was mentioned (see point 4 in subsection Background), all functions $f_{i j}(\mathcal{S})$ depend upon the behaviour of trajectory 
$S$ till time moment $j l$ (the end of subinterval $j$ ). In order to achieve this in our algorithm we should store all simulated earlier values $\mathcal{S}_{l}, \mathcal{S}_{2 l}, \ldots, \mathcal{S}_{j l}$, not only the last one as in previous examples.

The second issue is related to usings of procedure GenLossAmount (W). As we can see for each simulation this procedure is used $k$ times. In accordance with Section 4.1, we should use only one scenario from EDGE software for all these callings during each single of $m$ simulations. Of course, for every simulation the procedure GenLossAmount should be used with other scenario.

The last issue is connected with division of payments into constant part and nonconstant part, as in (32). The main reason is to achieve more transparency in the algorithm, e.g. the fact that procedure GenPaymentD needs no data from the behaviour of the trajectory $S$ is now easily seen.

Application This example is more general and complex than the one from Section 5.2. Because of this, not all the hints from Section 5.2 should be used also here. Especially it concerns the remarks about transferring the computional resources into more simulations rather than into more steps.

If the payment functions from matrix $\mathcal{P}_{f}$ depend only on the values of stochastic process $\mathcal{S}_{t}$ on the ends of subintervals, we have the same situation as in Section 5.2 and the appropriate remarks are still true. But the problem will be more complicated if we assume that some of functions $f_{i j}(\mathcal{S})$ need data from other time moments than $j l$ for $j=1, \ldots, k$. Such a problem is very similar to one connected with pricing American-style options. In this case we should consider very precisely how much computional time we may spend for simulations and how much for steps in each of simulations. And unfortunately there is no general rule in this case.

The second very important issue is that $\mathcal{S}_{t}$ and $\mathcal{X}_{t}$ are mutually independent stochastic processes. This assumption may be acknowledged as unrealistic from practical point of view. One may argue that the movements of interest rates and losses from catastrophes should be dependent, i.e. occurrence of catastrophe, especially very serious one, may have significant influence on the market. But the assumption of independency of $\mathcal{S}_{t}$ and $\mathcal{X}_{t}$ is usually implied in the discussion of cat bonds.

Nevertheless, resignation from this assumption has no impact on the method developed in this paper. In the first place, we may change the stochastic process $\mathcal{S}_{t}$ to other type which could model movements of interest rates with shocks from catastrophes. One example of such a process is geometrical Brownian motion with Poisson jumps (see Section 3.1.3). The choice of suitable process depends on the particular case study and we do not deal with it in this paper. For additional information see e.g. [10], [25].

An important issue is to take into account a possible dependence between processes. For example - the occurrence of triggering point may influence the behaviour of process $\mathcal{S}_{t}$. The application of Monte Carlo methods for such a case is possible and it requires only relatively small changes in algorithms. 


\section{Concluding remarks}

The main idea of the methodology presented in this paper is to use a combination of ISE (see Section 3.1.1) and Monte Carlo (see Section 3.2) methods for pricing the risk-transfer financial instruments. We present examples with algorithms in pseudocode of applying this methodology for the cat bonds depending on earthquakes (see Section 5).

In Section 3.3 we argue that cat bonds become important financial instruments nowadays, because of severity of natural catastrophes caused by climate changing (see Section 1). As we have noted losses and other undirected costs produced by huge floods, earthquakes and hurricanes have very huge negative impact on both macroeconomic and microeconomic scale.

In this case an important solution is to use new types of financial instruments - so called risk-transfer instruments, e.g. cat bonds. The problem connected with these instruments is calculation of their present value.

In practice such a calculation may be treated as the first necessary step before selling a new financial instrument. Knowledge of the present value of a given instrument is always the starting point for establishing its market price by the seller. In case of the lack of such an information, emission of this instrument may bring enormous losses for the prescriber.

In this paper we deal with this issue for the cat bonds depending on earthquakes.

However, the combination of Monte Carlo and ISE methods is very general and flexible and it may be used for variety of other cat bonds and different risk-transfer financial instruments.

Let us summarize some possible extensions to other types of risk-transfer financial instruments. The introduction of connection between the trajectory of underlying asset movements and the scenario of catastrophes (see Section 5.3) seems to be a very fruitful direction in the further researches. Such a dependence appears often in real-life examples.

The assumption of dependency between both processes requires appropriate model for $\mathcal{S}_{t}$. As we can see from literature the choice of this model is not so easy and straightforward (see e.g. [10]). But conceptually, after choosing an adequate stochastic process $\mathcal{S}_{t}$ there are no other barriers in applying ISE - Monte Carlo method.

The issue that all examples considered in Section 5 are connected with a very specific triggering point - an earthquake, is dictated by possibility of using procedures originated from EDGE software. However, it is easy to see that we can use variety of other types of triggering points - e.g. floods (see [19], [23]), hurricanes (like in A-1 bond, see Section 3.3 and [17], [28]). Cat bonds depending on such events may be very important for countries not suffering from earthquakes but rather from very serious floods (like Austria, Czech Republic, Germany, Poland, etc.). The only requirement in these cases is a necessity of using additional procedures for generating scenarios of appropriate catastrophe. Of course such procedures and software have to be relevant and meet needs of real-life application. Additionally, these procedures should be calibrated for the given region of interest.

At IIASA software similar to EDGE was developed for simulating scenarios of floods for Upper Tisza River basin (see [8]). It may be used in further researches or 
case studies.

There is also a possibility to generalize the model presented in this paper for other types of risk-transfer instruments (like contingent credit - see [8], [31]).

As we can see from this discussion the possibilities of using ISE - Monte Carlo methods vary widely and should be very fruitful in the future. Furthermore we should mention that the method presented in this paper is similar to the methodology widely used for other types of financial instruments, like options. This similarity may help to improve the reception of cat bond and other risk-transfer financial instruments by markets and experts in economy (see e.g. [2]). Therefore, it may contribute to wider usage of these instruments by new sellers in future.

\section{References}

[1] Baranov S., Digas B., Ermolieva T., Rozenberg V., "Earthquake Risk Management: A Scenario Generator", IIASA, IR-02-025, 2002

[2] Bantwal V. J., Kunreuther H. C., "A Cat Bond Premium Puzzle?", The Journal of Psychology and Financial Markets, 2000, Vol. 1, No. 1, pp. 76 - 91

[3] "Catastrophes, Infrastructure and Poverty", Options, IIASA, 1999

[4] "Climate change and Increase in Loss Trend Persistence", Press Release, Munich Re., Munich, 1999

[5] Consinglio A., Zenious S., "Designing Portfolios of financial products via integrated simulation and optimization models", University of Cyprus, Revised Report 96-05, 1998

[6] Cummins J. D., Doherty N., "Can Insurer Pay for the "Big One"? Measuring Capacity of an Insurance Market to Respond to Catastrophic Losses", working paper, Wharton Risk Management and Decision Processes Center, University of Pennsylvania, Philadelphia, PA, 1996

[7] Davis M., "Mathematics of Financial Markets" in Engquist B., Schmid W., "Mathematics Unlimited - 2001 \& Beyond", Springer Berlin 2001

[8] Ermolieva T., Ermoliev Y., Linnerooth-Bayer J., Galambos I., "The Role of Financial Instruments in Integrated Catastrophic Flood Management"

[9] Ermolieva T., Ermoliev Y., Norkin V., "Spatial Stochastic Models for Optimization Capacity of Insurance Networks Under Dependent Catastrophic Risks: Numerical Experiments", IIASA Interim Report, IR-97-028, 1997

[10] Ermoliev Y. M., Ermolieva T. Y., MacDonald G. J., Norkin V. I., "Catastrophic Risk Management and Economic Growth", in Youmin X., Haijun H., Liang L., Kanliang W. (eds) "New Management Trends in New Century", Proceedings of ICM'2001, May 2001, CHEP and Springer Verlag 
[11] Ermoliev Y. M., Ermolieva T. Y., MacDonald G. J., Norkin V. I., "Insurability of catastrophic risks: the stochastic optimization model", Optimization Journal, Vol. 47, 2000

[12] Froot K., "The Limited Financing of Catastrophe Risk: an Overview", Harvard Business School and National Bureau of Economic Research, 1997

[13] Freeman P. K., Pflug G. Ch., "Infrastructure in developing countries: Risk and Protection", May 22, 2001

[14] Galanti, Jang, "Low Discrepancy Sequences: Monte Carlo Simulation of Option Prices", Journal of Derivatives, Fall 1997

[15] George J. B., "Alternative reinsurance: Using catastrophe bonds and insurance derivatives as a mechanism for increasing capacity in the insurance markets", CPCU Journal, Spring 1999

[16] Gilks W. R., Richardson S., Spiegelhalter D. J., "Markov Chain Monte Carlo in Practice", Chapman \& Hall, 1997

[17] Hofmann M., "Cat bond market fears more red tape", Business Insurance, May 27,2002

[18] Hull J. C., "Options, Futures and Other Derivatives", Presntice Hall, 1997

[19] IPCC, "Climate Change 2001: Impacts, Adaption and Vulnerability", www.ipcc.ch

[20] Jobst N., Zenios S., "The Tail that Wags the Dog: Integrating Credit Risk in Asset Portfolios", Journal of Risk Finance, 2001

[21] Karatzas I., "Methods of mathematical finance", New York, Springer, 1998

[22] Korn R., Korn E., "Option Pricing and Portfolio Optimization", American Mathematical Society 2001

[23] Lizak K., draft paper about construction of FloodBond

[24] Lane M. N., Beckwith R. G., "Current Trends in Risk-Linked Securitization. March 2000 to March 2001", April 30, 2001, www.LaneFinancialLLC.com

[25] MacKellar L., Freeman P., Ermolieva T., "Estimating Natural Catastrophic Risk Exposure and the Benefits of Risk Transfer in Developing Countries", IIASA

[26] Matsumoto M., Nishimura T., "Mersenne Twister: A 623-dimensionally equidistributed uniform pseudorandom number generator", ACM Trans. on Modelling and Computer Simulation Vol. 8, No. 1, January, pp.3-30 1998, http://www.math.keio.ac.jp/ matumoto/emt.html

[27] Moro B., "The Full Monte", Risk, Vol. 8, No. 2, 1995 
[28] Niedzielski J., "USAA places catastrophe bonds", National Underwriter, Jun 16,1997

[29] Nowak P., "Analysis of applications of some ex-ante instruments for the transfer of catastrophic risks", IIASA, IR-99-075, 1999

[30] Nowak P., Nycz P., Romaniuk M., "Dobór optymalnego modelu stochastycznego w wycenie opcji metodami Monte Carlo" (in Polish), conference material on BOS 2002

[31] Pollner J., "Catastrophe Risk Management: Using Alternative Risk Financing and Insurance Pooling Mechanism, Finance, Private Sector $\&$ Infrastructure Sector Unit", Caribbean Country Department, Latin America and the Caribbean Region, World Bank 2000

[32] Robert C. P., Casella G., "Monte Carlo Statistical Methods", Springer Verlag 1999

[33] Rozenberg V., Ermolieva T., Blizorukova M., "Modelling earthquakes via computer programs", IIASA, IR-01-068, 2001

[34] Shiryaev A. N., Kruzhilin N., "Essentials of Stochastic Finance", World Scientific Publishing Co. Pte. Ltd., 1999/2000

[35] Walker G., "Current Developments in Catastrophe Modelling" in Britton N. R., Olliver J. (Eds.), Financial Risks Management for Natural Catastrophes, Brisbane, Griffith University, Australia, 1997 